\section{Exercise in water is beneficial for patients with fibromyalgia}

Two separate studies published in the journal Arthritis \& Rheumatism (Arthritis Care \& Research) have shown that patients with fibromyalgia could benefit from exercise in water. Regular physical exercise has proven to be beneficial in patients with fibromyalgia, but little is known about the specific effects of hydrotherapy. The two studies-one performed in Brazil and the other in Spain-aimed to evaluate the effect of water therapy on clinical aspects of fibromyalgia.

In the Brazilian study, the authors evaluated the efficacy of deep-water running (DWR) compared with that of land-based exercises (LBE) in 60 sedentary women, aged $18-60$ years, with fibromyalgia. In the DWR group $(n=30)$, patients performed a running movement while immersed in warm water $\left(28-31^{\circ} \mathrm{C}\right)$ up to shoulder level. In the LBE group $(n=30)$, patients walked or jogged around the local park. Both groups exercised for $1 \mathrm{~h}$, three times a week, for 15 weeks.

The study showed that the clinical endpoints of pain, mood, function, and quality of life improved in both the DWR and LBE groups to a similar degree. There was more improvement in the emotional aspects of fibromyalgia in the DWR group compared with the LBE group, although the authors caution that this study was not designed to assess this outcome. Four patients dropped out of the study, but not because of adverse events. There were no serious side effects in either group, confirming that patients with fibromyalgia can undergo physical training without worsening of symptoms.

In the Spanish study, the investigators evaluated the efficacy of exercise in waist-high, warm water $\left(33^{\circ} \mathrm{C}\right)$ in 34 women, aged 35 73 years, who had severe fibromyalgia. The 17 women assigned to the exercise group trained in water for $1 \mathrm{~h}$, three times a week for 12 weeks. At the end of the 12 weeks, the exercise group was instructed to avoid physical exercise for a further 12 weeks. A control group of 17 women followed their normal daily activities for 24 weeks.

In the exercise group, there were marked and clinically relevant gains in muscle strength of the knee extensors at low velocities, which is a major predictor of daily living dependency; however, there was no improvement at medium velocities, which is associated with gait speed. Improvements in muscle strength were maintained after the 12-week rest period. Pain, self care and depression improved after the 12 weeks of exercise, and most of these improvements were maintained; however, pain levels returned to baseline after 12 weeks of rest.

These two studies show that exercise in warm water is safe and effective, and is a viable form of low-impact exercise for patients with fibromyalgia. Since fibromyalgia is a chronic disease, studies to evaluate the long-term efficacy of exercise in water are warranted.

Rachel Murphy

Original articles Assis MR et al. (2006) A randomized controlled trial of deep water running: clinical effectiveness of aquatic exercise to treat fibromyalgia. Arthritis Rheum 55: 57-65

Gusi N et al. (2006) Exercise in waist-high warm water decreases pain and improves health-related quality of life and strength in the lower extremities in women with fibromyalgia. Arthritis Rheum 55: 66-73

\section{Increased lymphoma risk associated with high disease activity in rheumatoid arthritis}

On average, a rheumatoid arthritis (RA) patient's risk of lymphoma is twice that of the general population's. Moreover, an association has been noted between high levels of disease activity and lymphoma risk. Previous studies, however, have been unable to separate the risk posed by the disease itself from that posed by disease treatment. A Swedish team conducted a retrospective, casecontrolled study, investigating the effect of disease activity, RA phenotype, and treatment on lymphoma risk.

Baecklund et al. analyzed data from several population-based registers and biobanks in Sweden, covering the period 1964-1995. They found that disease activity was related to lymphoma risk: moderate disease activity was associated with an eightfold increase in risk compared with low disease activity, and high disease activity was associated with a 70-fold increase in risk. Treatment (including anti-inflammatory and disease-modifying antirheumatic drugs, methotrexate and steroids) did not increase lymphoma risk, with the exception of azathioprine. Use of this drug 